\title{
Comparação entre diferentes métodos de obtenção da razão sinal- ruído em imagens por ressonância magnética
} Comparison of different methods of obtaining the signal to noise ratio in magnetic resonance imaging

\author{
Briane E. S. Bandeira ${ }^{1}$, Jéssica V. Real ${ }^{1}$, Ana Paula P. Froner ${ }^{1}$, Ricardo M. Papaléo ${ }^{2}$, \\ Ana M. Marques da Silva ${ }^{2}$ \\ ${ }^{1}$ Serviço de Física Médica, Hospital São Lucas, PUCRS, Porto Alegre, Brasil \\ ${ }^{2}$ Faculdade de Física, PUCRS, Porto Alegre, Brasil
}

\begin{abstract}
Resumo
A literatura relata diferentes métodos para quantificar a razão-sinal-ruído ou SNR (Signal-to-Noise-Ratio) em imagens por ressonância magnética, de acordo com a bobina de radiofrequência utilizada. Este trabalho tem por objetivo apresentar a avaliação de dois métodos de medida da SNR baseadas na publicação da NEMA, variando-se a largura de banda de recepção $\left(B w_{r}\right)$ para uma sequência Spin Eco convencional. $\mathrm{O}$ método 1 , denominado subtração das imagens, resultou em uma curva cujo valor do parâmetro de interesse foi de $(-0,49 \pm 0,01) \mathrm{com} \mathrm{R}^{2}$ igual a 0,99664 . O método 2 , denominado rú́do do fundo, resultou em um valor de $(-0,50 \pm 0,01) \mathrm{com} \mathrm{R}^{2}$ igual a 0,99581 . Ambos os métodos se mostraram satisfatórios para a obtenção da SNR, não apresentando diferenças significativas com relação aos resultados.
\end{abstract}

Palavras-chave: ressonância magnética; razão-sinal-ruído; controle de qualidade; SNR.

\section{Abstract}

The literature describes different methods to measure the signal-to-noise ratio, or SNR, in magnetic resonance imaging, according to the radio frequency coil used. This paper aims to present the evaluation of two of the SNR measurement methods based on NEMA publication, varying the bandwidth of reception $\left(B w_{r}\right)$ in a Spin Eco conventional sequence. Method 1, called image subtraction, resulted in a curve whose parameter value of interest was $(-0.49 \pm 0.01)$ with $R^{2}$ equal to 0.99664 . The second method, called background noise, resulted in a value of $(-0.50 \pm 0.01)$ with $R^{2}$ equal to 0.99581 . Both methods were satisfactory to obtain SNR, no significant differences regarding the results.

Keywords: magnetic resonance; signal-to-noise ratio; quality control; SNR.

\section{Introdução}

A razão sinal-ruído ou SNR (Signal-to-NoiseRatio) é um parâmetro extremamente importante na avaliação da qualidade das imagens por ressonância magnética (RM) e pode ser utilizada a fim de comparar diferentes técnicas para obtenção de imagens ou como parte de um programa de garantia de qualidade.

Todas as medidas físicas incluem tanto o ruído aleatório quanto o sistemático, que podem afetar gravemente a precisão ou a interpretação de uma medida. O grau com que o ruído afeta uma medida é geralmente caracterizado pela SNR, um parâmetro chave para a determinação da eficácia de qualquer experimento de imagem. Se a SNR não é suficientemente alta, é impossível diferenciar um tecido de outro ou do fundo nas imagens por $\mathrm{RM}^{1}$.

A SNR depende de vários parâmetros, tais como o número de excitações da fatia, as dimensões do voxel $(\Delta x, \Delta y$ e $\Delta z)$, o número de amostras no espaço-k $\left(N_{x}, N_{y}\right.$ e $\left.N_{z}\right)$, a largura de banda de recepção $\left(B w_{r}\right)$, entre outros.

$A B w_{r}$ define a faixa de frequências à qual o detector é sintonizado durante a aplicação do gradiente de leitura na aquisição de imagens de RM. Uma largura de banda estreita, em torno da frequência central, fornece valores maiores de SNR, pois no domínio espacial a largura de banda é inversamente proporcional ao tempo de permanência da amostra. Portanto, uma largura de banda estreita tem um tempo de permanência mais longo, o que, por consequência, aumenta a intensidade do sinal em comparação com uma largura de banda mais larga, que espalha o sinal ao longo de um intervalo maior de frequências ${ }^{2}$.

Há na literatura diferentes métodos para quantificar a SNR, de acordo com a bobina de radiofrequência utilizada. Sabe-se que a SNR é inversamente proporcional à raiz quadrada da $\mathrm{Bw}_{\mathrm{r}}{ }^{2}$ e que a $\mathrm{Bw}_{\mathrm{r}}$ altera significativamente os valores de ruído da imagem ${ }^{3}$.

O objetivo desse estudo é investigar, através da relação conhecida entre a SNR e a $B w_{r}$, os diferentes métodos de determinação da SNR sugeridos pela publicação da NEMA (do inglês National Eletrical Manufacturers Association $)^{[4]}$ para bobinas de volume de um único canal em imagens por RM. 


\section{Materiais e Métodos}

Para determinar a SNR, a NEMA recomenda que 0 objeto de teste possua algumas características, tais como o tempo de relaxação longitudinal $\left(T_{1}\right)$ menor do que $1200 \mathrm{~ms}$ e de relaxação transversal $\left(\mathrm{T}_{2}\right)$ maior do que $50 \mathrm{~ms}$.

O objeto de teste é um simulador esférico $G E$ MRI Phantom modelo 46-265826G6 (Figura 1), com diâmetro de $(18,00 \pm 0,05) \mathrm{cm}$.

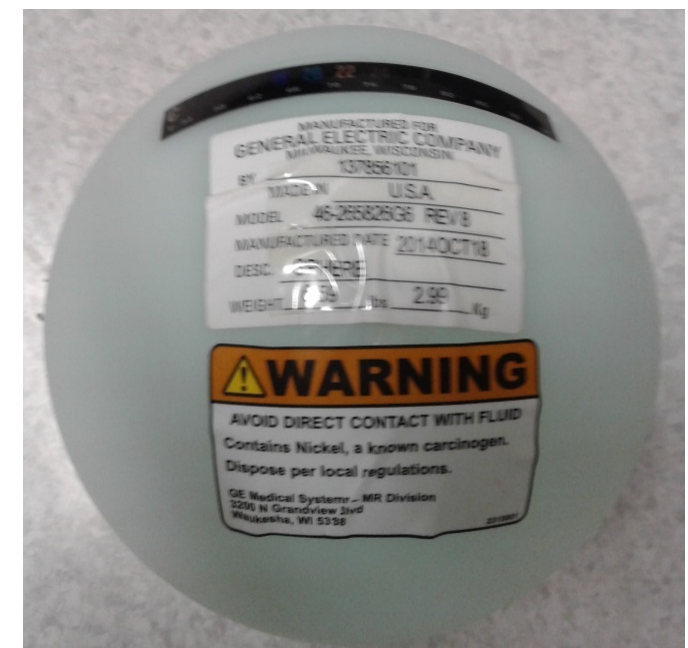

Figura 1. Objeto de teste esférico GE MRI Phantom modelo 46-265826G6.

Inicialmente foi adquirida uma imagem do objeto de teste a fim de encontrar os valores de $T_{1}$ e $T_{2}$.

O objeto de teste foi centralizado na bobina de radiofrequência e a bobina foi posicionada no isocentro do equipamento. As imagens de RM foram adquiridas em um equipamento da marca GE HealthCare, modelo BRIVO 1,5 T 355, com a bobina de crânio modelo Split Head Coil.

Para obtenção de $T_{1}$, uma sequência Spin Eco IR (Inversion Recovery) foi utilizada e várias imagens foram adquiridas com diferentes tempos de inversão (TI). Nesse método de obtenção de $\mathrm{T}_{1}$, a magnetização longitudinal $\left(\mathrm{M}_{\mathrm{z}}\right)$ pode ser escrita na forma:

$$
M_{z}=A+B e^{-\left(\frac{T_{I}}{T_{1}}\right)}
$$

onde $A$ e $B$ são constantes, $T_{1}$ e $T_{1}$ são os tempos de inversão e de relaxação longitudinal, respectivamente ${ }^{5}$.

Para obtenção de $T_{2}$ foi utilizada uma sequência Spin Eco variando-se o tempo de eco $\left(T_{E}\right)$ e fixando-se os demais parâmetros. Nesse caso, a magnetização transversal $\left(\mathrm{M}_{\mathrm{xy}}\right)$ pode ser escrita como:

$$
M_{x y}=A e^{-\left(\frac{T_{E}}{T_{1}}\right)}
$$

Após a obtenção dos valores de $T_{1}$ e $T_{2}$ do objeto de teste, foram definidos os parâmetros de aquisição das imagens a fim de avaliar a SNR por diferentes métodos. Outra recomendação da NEMA é que o valor do tempo de repetição $\left(T_{R}\right)$ da sequência Spin Eco seja pelo menos três vezes o valor de $\mathrm{T}_{1}$ do material produtor de sinal do objeto de teste. Os parâmetros utilizados na aquisição das imagens encontram-se na Tabela 1.

$\begin{aligned} & \text { Tabela 1. Parâmetros de aquisição das imagens para } \\
& \text { determinação da SNR. }\end{aligned}$
\begin{tabular}{ll}
\hline Sequência & Spin Eco \\
\hline Plano & Axial \\
FOV $(\mathrm{cm})$ & 30 \\
Phase FOV & 1 \\
Espessura de corte $(\mathrm{mm})$ & 2 \\
Espaçamento $(\mathrm{mm})$ & 1,5 \\
Direção da Frequência & $\mathrm{S} / \mathrm{l}$ \\
TR (ms) & 600 \\
Número de cortes & 2 \\
TE por scan & 1 \\
TE (ms) & 20 \\
Frequência & 256 \\
Fase & 256 \\
NEX & 1
\end{tabular}

Antes de adquirir as imagens, foi realizado um autoprescan a fim de obter o valor da frequência central $(\mathrm{MHz})$. Posteriormente, uma sequência Spin Eco convencional foi utilizada obtendo imagens com diferentes valores de $B w_{r}$. Sempre que se varia um parâmetro em $R M$, os efeitos resultantes sobre os demais parâmetros devem ser checados.

Em todos os métodos descritos, o valor médio do sinal dentro do objeto simulador foi obtido através do software ARYA e com uma ROI (do inglês Region of Interest) que corresponde a $75 \%$ da área desse objeto.

A seguir estão descritos os dois métodos de determinação da SNR.

\subsection{Método 1: Subtração das Imagens}

O método 1 exige a subtração de duas imagens adquiridas consecutivamente, com os mesmos parâmetros, gerando uma terceira imagem, da qual se obtém o valor do desvio padrão ou SD (do inglês Standard Deviation). Esse procedimento de subtração e obtenção do SD foi realizado através do software livre ImageJ.

O valor de SD na terceira imagem foi obtido com uma ROI igual a $75 \%$ da área do objeto de teste a partir da imagem subtraída, conforme ilustra a Figura 2.

onde A é uma constante. ${ }^{[5]}$ 


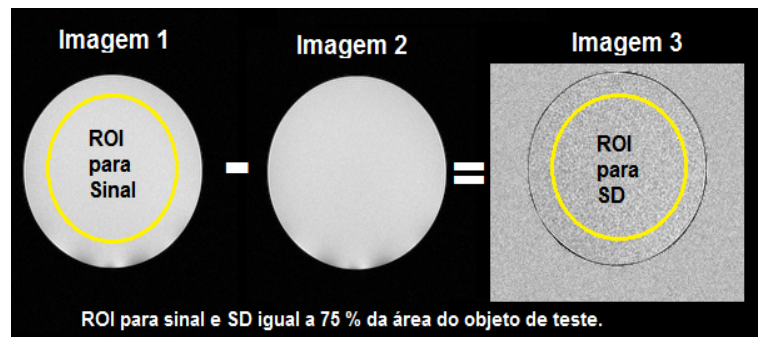

Figura 2. Obtenção do Sinal e do SD de acordo com o método 1.

Como o valor de SD é obtido de uma imagem subtraída, o fator $\sqrt{2}$ é necessário para compensar o maior desvio padrão da subtração das imagens ${ }^{3}$. O ruído é então obtido através da relação:

$$
\text { Ruído }=\frac{S D}{\sqrt{2}}
$$

A SNR nesse método é dada por:

$$
S N R_{\text {método } 1}=\sqrt{2} \frac{\text { Sinal }}{S D}
$$

\subsection{Método 2: Ruído do fundo}

No método 2, o valor do SD é obtido com ROls compostas por, pelo menos, 1000 pixels, localizadas nos cantos da imagem, distantes do objeto de teste e livres de quaisquer artefatos visíveis, conforme ilustra a Figura 3.

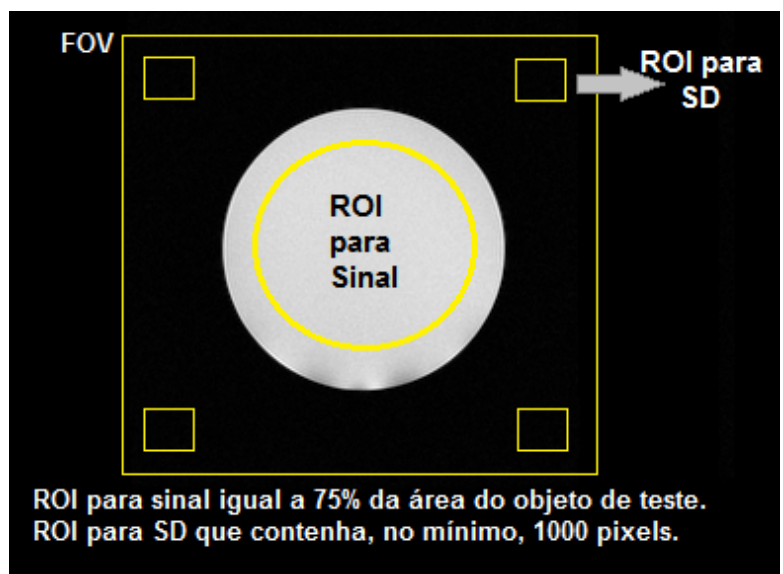

Figura 3. Obtenção do Sinal e do SD de acordo com o método 2 .

Nesse método, o SD no fundo da imagem foi corrigido pelo fator 0,66 , pois o ruído de fundo segue uma distribuição de Rayleigh ${ }^{3}$.Assim, o ruído na imagem é dado pela equação 5 :

$$
R u i ́ d o=\frac{\overline{S D}}{0,66}
$$

A SNR nesse método é dada por:

$$
S N R_{\text {método } 2}=0,66 \frac{\text { Sinal }}{\overline{S D}}
$$

Em ambos os métodos, uma curva é ajustada aos dados usando software Origin 7.0, de acordo com a equação 7 :

$$
\mathrm{SNR}=a\left(B w_{r}\right)^{b}
$$

\section{Resultados}

A Figura 4 mostra o gráfico do sinal obtido em cada imagem, em função do seu respectivo tempo de inversão, para obtenção do $T_{1}$ do objeto de teste.

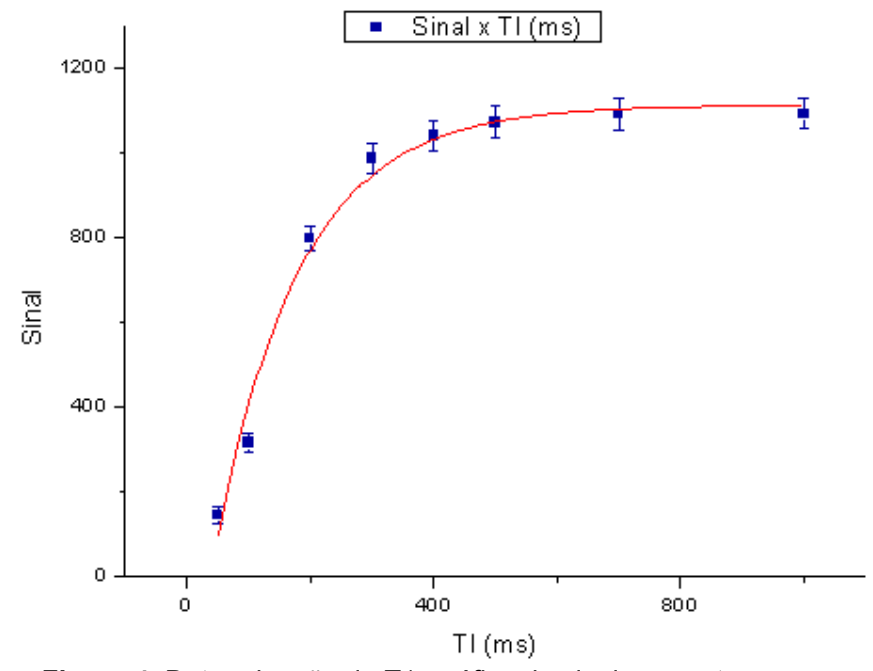

Figura 4. Determinação de T1: gráfico de sinal versus tempo de inversão (TI).

De acordo com a equação 1 , o ajuste da curva resultou em um valor de $T_{1}$ de $(138 \pm 19) \mathrm{ms}$ com $R^{2}$ igual a 0,9869 .

A Figura 5 mostra o gráfico do sinal obtido em cada imagem, em função do seu respectivo tempo de eco, para obtenção do $T_{2}$ do objeto de teste.

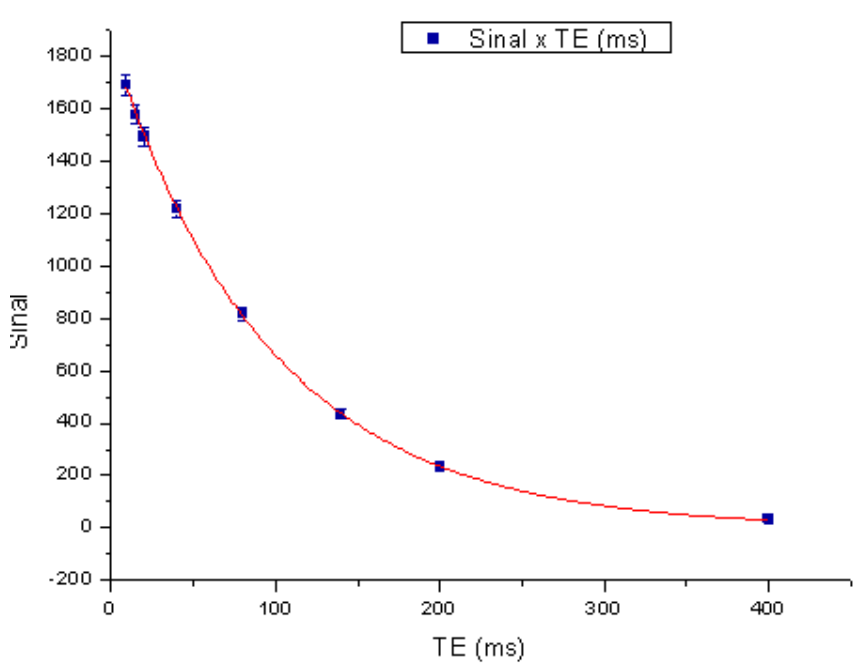

Figura 5. Determinação de T2: gráfico de sinal versus tempo de eco (TE).

De acordo com a equação 2 , o ajuste da curva resultou em um valor de $T_{2}$ de $(97,1 \pm 0,6) \mathrm{ms}$ com $R^{2}$ igual a 0,99993 .

onde $a$ e $b$ são constantes. 
A Figura 6 mostra o gráfico de SNR $\times \mathrm{Bw}_{\mathrm{r}}$ para os dois métodos analisados.

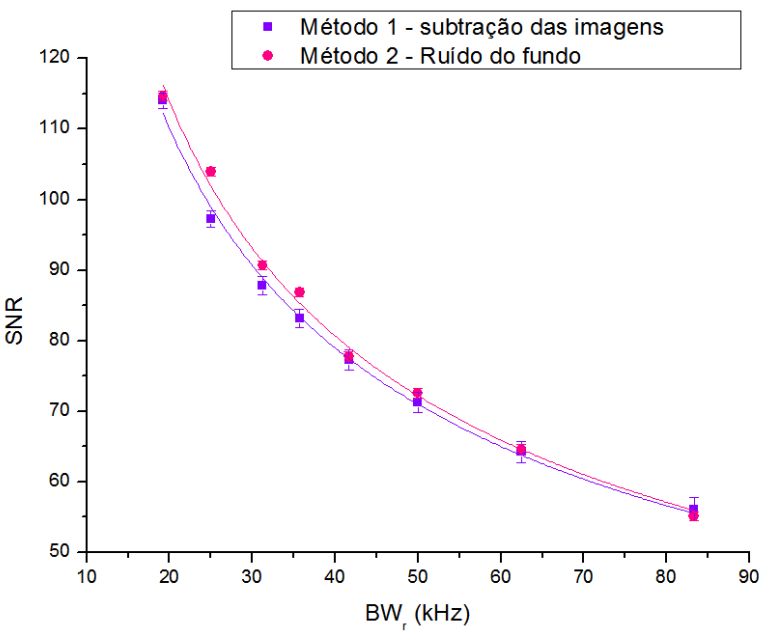

Figura 6. Gráfico de SNR $\times$ BWr $(\mathrm{kHz})$ para os dois métodos de determinação da SNR.

De acordo com a equação 7 , o ajuste da curva resultou para o método 1 (subtração das imagens) em $b=-0,49 \pm 0,01$ com $R^{2}$ igual a 0,99664 e para o método 2 (Ruído do fundo) em -0,50 $\pm 0,01 \mathrm{com}$ $\mathrm{R}^{2}$ igual a 0,99581 . O valor da frequência central obtido antes da aquisição das imagens foi de 63,87 $\mathrm{MHz}$ para o equipamento de RM de 1,5 tesla.

\section{Discussão}

Através dos resultados nota-se que os dois métodos de determinação da SNR propostos pela NEMA não diferem significativamente em seus resultados. O método 1 mostra um valor de $R^{2}$ mais próximo de 1 do que o método 2 , o que significa que os seus dados se ajustam ligeiramente melhor à curva.

O método 1 difere em $2 \%$ com relação ao valor esperado do parâmetro $b$ (igual a $-0,5$ ), enquanto que o método 2 resulta exatamente nesse valor.

\section{Conclusões}

Este trabalho comparou, através da relação conhecida entre a SNR e a Bwr, os diferentes métodos de determinação da SNR para bobinas de radiofrequência em imagens por $\mathrm{RM}$, segundo recomendações da NEMA.

Conclui-se que a escolha do método não interfere de forma significativa no valor resultante da SNR. No entanto, deve-se destacar que, para um programa de garantia de qualidade em imagens por ressonância magnética, é importante que o monitoramento da SNR seja sempre realizado sob as mesmas condições, tanto de aquisição, quanto de análise, garantindo que os valores sejam comparados à linha de base e se reproduzam de forma satisfatória.

\section{Referências}

1. HAACKE EM, et al. Magnetic Resonance Imaging - Physical Principles and Sequence Design. New Jersey: John Wiley \& Sons; 2014.
2. BUSHBERG JT, et al. The Essential Physics of Medical Imaging. Philadelphia: LIPPINCOTT WILLIAMS \& WILKINS, 2012.

3. MCROBBIE DW, et al. MRI from Picture to Proton. New York: Cambridge University Press; 2006.

4. NEMA Standards Publication MS 1-2008 (R2014). Determination of Signal-to-Noise Ratio (SNR) in Diagnostic Magnetic Resonance Imaging.

5. TOFTS PS. Methods for quantitative relaxation parameter mapping: measuring T1 and T2. ISMRM. 18 de Abril de 2009, Hawaii. Disponivel em: http://www.paul-toftsphd.org.uk/talks/ismrm2009 rt.pdf. Acesso em: 20 de Abril de de 2016

\section{Contato:}

Briane E. S. Bandeira

Hospital São Lucas da PUCRS, Av. Ipiranga 6690, CEP 90610-000, Porto Alegre, RS.

E-mail: briane.bandei@pucrs.br 